\title{
There and Back Again. . Safely: Examining Students' Reports of Substance Use and Sexual Assault Prevention Program Receipt Prior to Departure Abroad
}

\author{
Sierra Smucker \\ RAND Corporation
}

Eric R. Pedersen

RAND Corporation

Joseph W. LaBrie

Loyola Marymount University

Elizabeth J. D'Amico

RAND Corporation

Coreen Farris

RAND Corporation

David J. Klein

RAND Corporation

\section{Beth Ann Griffin}

RAND Corporation

\begin{abstract}
:
Many universities have pre-departure programs that aim to prevent alcohol misuse and sexual assault among college students abroad, yet little is known about students' uptake of this preventative information. In this study, 2,245 study abroad students from 63 different institutions were asked about receiving pre-departure alcohol/drug and sexual assault prevention information. Only 38\% of students reported receiving pre-departure information specific to alcohol/drug misuse abroad and only $22 \%$ reported receiving sexual assault prevention information prior to departure. Notably, students who were heavier drinkers prior to departure and those who intended to drink the most while abroad reported lower rates of information receipt. Overall, these findings suggest that either students are not receiving the preventative information that universities intend them to receive or the programming is not impactful enough to be recalled. Thus, there may be missed opportunities to better prepare students for risks specific to study abroad programs.
\end{abstract}

\section{Introduction}

Study abroad is an increasingly common experience for American undergraduate college students. Over 330,000 students studied abroad during the 2016/2017 school year, which represents 
a nearly $30 \%$ increase in the number of students over the past 10 years and over a $220 \%$ increase over the past 20 years (Institute of International Education, 2018). As increasing numbers of students participate in study abroad programs, it is important to understand the potential for risky behaviors by students abroad and whether program directors are doing enough to address those risky behaviors.

One of the risks of study abroad is that many students substantially increase their alcohol use while abroad compared to their use at their home institution (Aresi, Moore, \& Marta, 2016). For example, one study found that U.S. college students doubled their drinking while abroad (Pedersen, Larimer, \& Lee, 2010). Drinking abroad can often result in negative consequences for students, including passing out, blacking out, and alcohol-related injuries (Hummer, Pedersen, Mirza, \& LaBrie, 2010; Pedersen, Neighbors, Lee, \& Larimer, 2012).

Students studying abroad may also face a higher risk of sexual assault than their peers at their home institution. For example, one study of 218 female undergraduates found that the students were three to five times more likely to experience sexual assault while abroad than on their home campus (Kimble, Flack, \& Burbridge, 2013). A similar study of 2018 female students at the same institution found that nearly 1 in 5 students (18.8\%) reported sexual assault victimization abroad, with non-consensual sexual touching being most prevalent (16.8\%) (Flack et al., 2015). Additionally, $6.7 \%$ of the sample reported attempted rape and 3.8\% reported completed rape. Moreover, the majority of student participants $(78.8 \%)$ were only studying abroad for a single semester or a shortterm summer program (i.e., one to three months), which suggests that students may experience these events during even brief periods of time abroad.

To minimize students' risk of alcohol misuse and sexual assault while abroad, program directors have developed a variety of resources including in-person meetings, online content, and information packets to provide students with relevant health and safety information before departure. However, there is limited research investigating the content of these programs and whether they include sexual risk and alcohol misuse prevention.

Marcantonio and co-authors (2018c) performed a content analysis of 753 U.S. study abroad program websites and found that most programs provided limited information about risky sexual behaviors or alcohol use on their websites. Only $17 \%$ of the sites provided information about risky sexual behavior, 26\% mentioned alcohol use, and 20\% provided information about sexual assault. However, website information may not reflect the entire body of information provided to students. Pre-departure orientations and trainings are a common method for communicating to students the unique risks of studying abroad as well as the protective behaviors students can adopt to avoid those risks. A survey of 136 program directors found that $85 \%$ reported that their pre-departure meetings with students included information about alcohol use and the associated risks. Further, 62\% reported that they addressed the risks of sexual assault specific to study abroad with students during pre-departure meetings (Marcantonio, Angelone, Swirsky, \& Joppa, 2018b).

Although these results show what material program directors provide students, no research to date has examined the student perspective. Directors may be offering safety information, but it is unclear if students engage with or remember this information. The present study fills this gap by focusing on how students received information about risks prior to studying abroad. This study 
leverages a unique dataset of survey responses from 2,245 students studying abroad in 2018 from 63 different U.S. colleges and universities.

\section{Methods}

\section{Participants}

Prospective study abroad students were recruited from 63 different U.S. colleges and universities to participate in a longitudinal study to examine drinking and sexual risk among college students abroad. Directors of study abroad offices at these institutions informed students who were registered for an upcoming study abroad program about the survey research study. Interested students completed an online screening questionnaire to determine eligibility based on the following criteria: (1) between the ages of 18 and 25, (2) planning to study abroad in one of the 12 most popular study abroad destinations (i.e., the United Kingdom, Italy, Spain, France, Germany, China, Ireland, Australia, Costa Rica, Japan, South Africa, and Mexico; representing 59\% of study abroad students), and (3) planned to study abroad for between 4 and 21 weeks, which represents about twothirds of all study abroad students (Institute of International Education, 2018). Students were recruited from three sequential cohorts: spring 2018, summer 2018, and fall 2018.

Of the 4,086 total students who expressed interest in the study, 2,692 met the inclusion criteria and were emailed a link to an online survey within two weeks of their departure date. Among the students that were invited to participate, 2,259 completed the survey; four of these surveys were duplicates, eight students decided not to study abroad after completing the survey, and two decided not to participate in the study after completing the survey. Thus, the final survey sample included 2,245 students. Participants gave consent online prior to beginning the survey, and they received a $\$ 20$ gift card for completing the survey. All procedures were approved by the RAND Institutional Review Board.

\section{Measures}

Demographics.

Participants indicated their sex, gender (man, woman, do not identify with a gender), age, race and ethnicity, class year, and the country of study while abroad. Items for sex and gender were used to generate a gender variable based on identified gender. Class year was dichotomized into upperclassmen (juniors/seniors) and underclassmen (freshmen/sophomores). Students indicated the college or university they attended, which we categorized based on size of undergraduate enrollment: small (less than 5,000 students), medium (between 5,000 and 15,000 students), and large (more than 15,000 students).

\section{Substance use programs.}

Participants responded to several questions about whether they had completed any alcohol or drug prevention programs during college, including a class, a meeting in their dorm, or an online program (yes/no response). Those who indicated they had completed such a program were asked whether the program they received was (a) mandatory for everyone at their institution (e.g., a program conducted during orientation), (b) required because they had violated school alcohol or drug policies, or (c) voluntary (e.g., as part of a research study being conducted on campus). Next, all participants were asked if they had completed any alcohol or drug prevention program specific to 
drinking risk abroad (yes/no response). For those that indicated they had completed such a program, they were asked whether the program was (a) mandatory for everyone at their institution (e.g., a program conducted during orientation), (b) required because they had violated school alcohol or drug policies, or (c) voluntary (e.g., as part of a research study being conducted on campus).

Sexual assault programs.

Participants were asked whether they had completed any sexual assault programs during college, such as a class, a meeting in their dorm, or an online program (yes/no response). All participants were then asked if they had completed any sexual assault program specific to sexual assault risk abroad (yes/no response). Students who indicated they had completed a sexual assault program specific to study abroad were then asked if the role of alcohol was discussed as a risk factor for sexual assaults during the program. Participants could respond with "no," “yes," or "I don't remember." Responses of "no" and "I don't remember" were combined for comparisons between those who completed a program and those who did not or did not remember.

Alcohol use.

Alcohol use was assessed via two measures for past 30 day use (pre-departure drinking) and intended drinking while abroad. Standard drinks were defined for participants prior to drinking questions, with examples of standard drinks for beer, wine, liquor, and mixed drinks.

Pre-departure drinking. Participants completed the Daily Drinking Questionnaire (DDQ) for the past 30 days (Collins, Parks, \& Marlatt, 1985). The DDQ asks participants to consider a typical week during the last month and indicate the number of drinks they typically consumed on each day of that week. Values for all seven days were summed to reflect a total number of drinks per week. Participants also reported the number of drinks they consumed in one night on any occasion in the past month when they drank the most (peak drinks).

Intended drinking abroad. Using a modified DDQ, participants indicated how many drinks they thought they would drink on each day of a typical week while abroad. Values for all seven days were summed to reflect a total intended drinks per week. Participants also reported the maximum number of drinks they thought they would drink in one night while abroad (intended peak drinks).

\section{Analytic Plan}

Our analyses consisted of descriptive statistics for sample characteristics and self-reported receipt of alcohol and sexual assault programming specific to study abroad. We conducted chisquared tests and Fisher's exact tests to examine whether reporting receipt of specific programs was associated with gender, class standing, and school size. We also conducted mean comparisons to assess whether there were differences between those who reported that they completed programs versus those who said they did not complete programs on pre-departure drinking levels and predeparture drinking intentions while abroad.

\section{Results}

\section{Student Demographics}

Table 1 details demographics of the sample. Students were 20 years old on average. Sixty-nine percent of students identified as white, while 14\% identified as Hispanic/Latino, $10 \%$ as Asian, 3\% as Black, and $5 \%$ as either multi-racial or American Indian/Alaskan Native. The sample included 
more women $(77 \%)$ than men $(22 \%)$; 7 students $(0.3 \%)$ did not identify with a gender. Twenty percent of students were first-year students or sophomores, with most $(80 \%)$ being juniors or seniors. Students came from a range of schools including small schools with less than 5,000 students $(13 \%)$, medium schools with 5,000 to 15,000 students $(31 \%)$, and large schools with more than 15,000 students $(56 \%)$.

Table 1. Sample descriptives.

\begin{tabular}{|c|c|c|}
\hline & \multicolumn{2}{|c|}{ Study Abroad Students $(N=2,245)$} \\
\hline & N/Mean & $\% / \mathrm{SD}$ \\
\hline Age & 20.10 & 0.95 \\
\hline \multicolumn{3}{|l|}{ Race/ethnicity } \\
\hline White & 1,539 & $68.6 \%$ \\
\hline Hispanic/Latino & 308 & $13.7 \%$ \\
\hline Asian & 235 & $10.5 \%$ \\
\hline Multi-racial & 102 & $4.5 \%$ \\
\hline Black & 58 & $2.6 \%$ \\
\hline America Indian/Alaskan Native & 3 & $0.1 \%$ \\
\hline \multicolumn{3}{|l|}{ Gender } \\
\hline Men & 502 & $22.4 \%$ \\
\hline Women & 1736 & $77.3 \%$ \\
\hline Does not identify with a gender & 7 & $0.3 \%$ \\
\hline \multicolumn{3}{|l|}{ School size } \\
\hline Small $(<5,000)$ & 283 & $12.6 \%$ \\
\hline Medium $(5,000-15,000)$ & 702 & $31.3 \%$ \\
\hline Large $(>15,000)$ & 1260 & $56.1 \%$ \\
\hline \multicolumn{3}{|l|}{ Class year } \\
\hline First-year/sophomore & 457 & $20.4 \%$ \\
\hline Junior/senior & 1788 & $79.6 \%$ \\
\hline \multicolumn{3}{|l|}{ Country of study } \\
\hline Australia & 214 & $9.5 \%$ \\
\hline China & 115 & $5.1 \%$ \\
\hline Costa Rica & 40 & $1.8 \%$ \\
\hline France & 188 & $8.4 \%$ \\
\hline Germany & 119 & $5.3 \%$ \\
\hline Ireland & 142 & $6.3 \%$ \\
\hline Italy & 399 & $17.8 \%$ \\
\hline Japan & 78 & $3.5 \%$ \\
\hline Mexico & 22 & $1.0 \%$ \\
\hline South Africa & 47 & $2.1 \%$ \\
\hline Spain & 500 & $22.3 \%$ \\
\hline United Kingdom $^{1}$ & 381 & $17.0 \%$ \\
\hline
\end{tabular}

Current and intended alcohol use 


\begin{tabular}{lll} 
Drinks per week past 30 days & 4.87 & 6.24 \\
Peak drinks past 30 days & 5.25 & 3.33 \\
Intended drinks per week abroad & 7.05 & 7.34 \\
Intended peak drinks abroad & 4.67 & 3.29 \\
\hline
\end{tabular}

${ }^{1}$ United Kingdom included study locations in England, Scotland, Wales, and Northern Ireland.

The racial and ethnic identity of the study population largely matched that of study abroad students at the national level and the sample contained a slightly higher proportion of women and upperclassmen than the national population of study abroad students $(71 \%$ white, $67 \%$ women, and $60 \%$ juniors or seniors; Institute of International Education, 2018). It should be noted that our sample was restricted by age, location of study abroad, and time period abroad, which may explain some of the differences between our sample and the full population of study abroad students.

The most popular destination for our sample was Spain $(22 \%)$ followed by Italy $(18 \%)$, the United Kingdom (17\%), Australia (10\%), France (8\%), Ireland (6\%), China (5\%), and Germany $(5 \%)$. The remaining students planned to study in Japan (3\%), Costa Rica (2\%), South Africa (2\%), and Mexico (1\%). Though our sample was restricted to students studying in these countries, the distribution of students per country matched the study abroad population, with Spain, Italy, and the United Kingdom the most popular study abroad destinations (Institute of International Education, 2018).

\section{Alcohol Use}

On average, student participants reported consuming 4.9 drinks per week, with 7.3 drinks per week for men and 4.2 drinks per week for women. Average peak drinking on one night was 5.3 drinks overall, with 7.2 drinks for men and 4.7 drinks for women. Students said that they intended to consume an average of 7.0 drinks per week, with men intending to drink 9.8 drinks per week and women intending to drink 6.3 drinks per week. The students said they intended to drink a maximum of 4.7 drinks on their peak drinking occasion, with 6.4 drinks for men and 4.2 drinks for women.

\section{Substance Use Programs}

The majority of participants (86\%) reported completing an alcohol or drug program at some point during college. Among those participants that attended such programming, most (97\%) reported that it was mandatory for all students at their institution. Six percent reported that they received a program that was mandatory due to an alcohol or drug-related violation, and 5\% said they received a program because they volunteered for it. Although most students completed alcohol or drug programs at some point in college, only $38 \%$ of the sample reported completing programs specific to the risks of drinking abroad. Of those who did complete a study abroad-specific alcohol and drug program, nearly all reported that it was mandatory at their institution (98\%). Less than 1\% reported the alcohol and drug program was mandatory due to an alcohol- or drug-related violation, and $3 \%$ reported they received a program because they volunteered for it.

There were no significant differences in the completion of study abroad-specific alcohol or drug programs based on either gender or class year. There was a significant difference in whether students completed a study abroad-specific alcohol or drug program based on school size, with 
students attending small schools less likely to report completion of programming $(33 \%)$ than students attending medium $(41 \%)$ or large $(38 \%)$ schools, $\mathrm{X}^{2}(d f=2)=6.08, p<.05$.

Table 3 shows that students who did not attend a study abroad-specific alcohol or drug program reported a greater number of pre-departure drinks per week and past 30 day peak drinks, and a greater number of intended drinks per week abroad and intended peak drinks abroad.

\section{Sexual Assault Programs}

The majority of participants (84\%) reported completing a sexual assault program at some point during college, but as shown in Table 2 , only $22 \%$ of the sample reported completing a program specific to the risks of sexual assault abroad. Of those who did complete study abroad-specific sexual assault prevention programming, most (80\%) reported that alcohol was discussed as a risk factor for sexual assault.

Table 2. Frequencies of students reporting they completed substance use and sexual assault programs specific to risk abroad in college.

\begin{tabular}{ll}
\hline & Percentage and number reporting "yes" \\
\hline $\begin{array}{l}\text { Completed an alcohol or drug program in college specific to drinking risk study } \\
\text { abroad }\end{array}$ & $\%(\mathrm{~N})$ \\
$\quad \begin{array}{l}\text { Mandatory program for all students } \\
\text { Mandatory program due to violation }\end{array}$ & $98.0 \%(859)$ \\
$\quad{ }^{\mathrm{a}}$ & $0.4 \%(3)$ \\
\hline $\begin{array}{l}\text { Completed a sexual assault prevention program specific to sexual assault risk } \\
\text { abroad }\end{array}$ & $3.4 \%(29)$ \\
$\quad$ Role of alcohol as a risk factor for sexual assault abroad was discussed & $21.9 \%(490)$ \\
\hline $\begin{array}{l}\text { a } \% / N \text { reported is among those who endorsed completing an alcohol or drug program specific to abroad. } \\
\text { b \%/N reported is among those who endorsed completing a sexual assault prevention program specific to abroad. }\end{array}$
\end{tabular}

There were no significant differences in whether students completed study abroad-specific sexual assault prevention programs based on gender or class year. There was a significant difference in reported completion of an abroad-specific sexual assault program based on school size, with students attending large schools less likely to report completion of programming (18\%) than students attending small $(26 \%)$ or medium $(28 \%)$ schools, $X^{2}(d f=2)=29.79, p<.001$. There were no significant differences in means for typical pre-departure drinks per week between those who completed study abroad-specific sexual assault prevention programs and those who did not. However, students who did not report completing a study abroad-specific sexual assault program reported greater past 30 day peak drinks, more intended typical drinks per week abroad, and greater intended peak drinks abroad than those who reported completing a study abroad-specific program (see Table 3). 
Sierra Smucker et al.

Table 3. Comparisons for drinking abroad factors between those who reported completed alcohol or drug programs and those who did not.

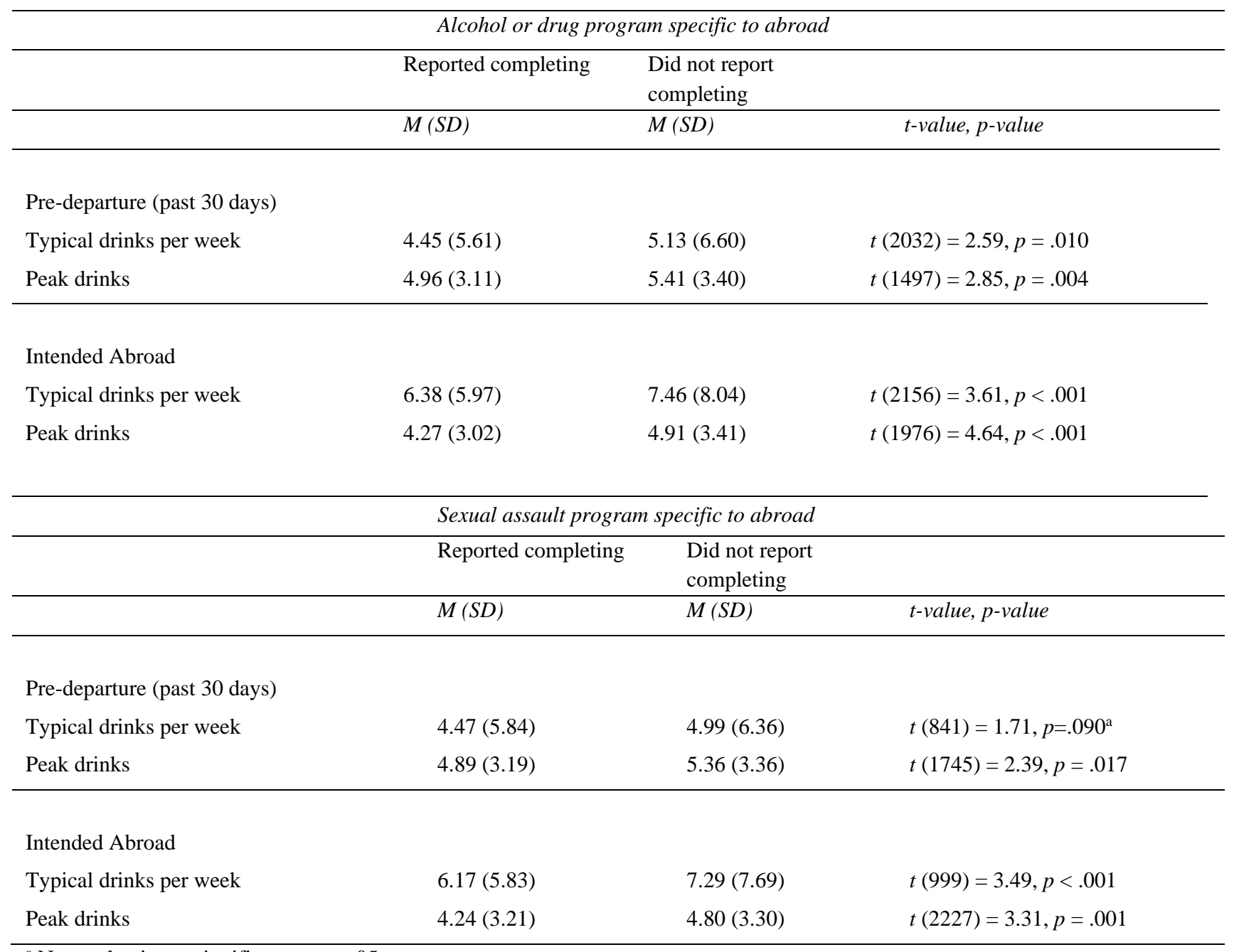

${ }^{a}$ Note value is not significant at $p<.05$

\section{Discussion}

Students studying abroad may face increased risk of alcohol misuse and sexual assault while away from their home institutions (Flack et al., 2015; Hummer et al., 2010; Kimble et al., 2013; Pedersen et al., 2010; 2012), and many study abroad program directors offer students pre-departure programs with preventive information related to alcohol misuse and risks of sexual assault as part of going abroad (Marcantonio et al., 2018b). However, no study to date has asked students whether they complete these programs before going abroad. This study is the first to address studentreported exposure to general pre-departure programming with preventive information specific to study abroad alcohol misuse and sexual assault.

Most students reported completing alcohol misuse and sexual assault risk reduction programs at some point during college, but most also indicated that they did not complete pre-departure programming specific to the risks of drinking and sexual assault while studying abroad. Risk 
reduction programs received earlier in students' college careers could possibly carry over to later years and be applied to one's time abroad, thereby producing a protective effect against alcohol misuse and sexual assault while abroad. However, the study abroad environment presents a new and particularly risky context for students as it introduces a host of unique risks. For example, students may face limited access to resources and familiar coping strategies, disrupted travel plans or injuries, unfamiliarity with local healthcare locations or law enforcement policies, and for sexual assault specifically, different sexual norms between countries and potential language barriers that interfere with sexual communication. All factors could lead to higher risk situations. Abroad-specific programing is particularly important given the increase in student drinking levels while abroad (Aresi et al., 2016; Pedersen et al., 2010) and the potential increased risk for sexual assaults during study abroad experiences (Fisher, Daigle, \& Cullen, 2009; Flack et al., 2015; Kimble et al., 2013; Marcantonio et al., 2016). Thus, many students are not receiving programming that addresses the risks of being abroad and that provides strategies for how to keep themselves safe.

Importantly, our findings highlight that heavier drinkers were significantly less likely to report completing a study abroad-specific alcohol or drug program. These students reported drinking significantly more before departure and they intended to drink significantly more frequently and heavily while abroad than students who completed a study abroad-specific program. Thus, heavier drinkers were less likely to complete an abroad-specific program than their counterparts despite their potential greater need for it. It is presumed that institutions are not withholding programing specifically from heavy drinkers, but it is possible that heavy drinking students may be more likely to skip sessions or forget to attend because of their heavy drinking habits. It is also possible that heavier drinkers tend to come from institutions that do not offer programs to any students. These results suggest that program directors may need to provide additional incentives to ensure those with heavy drinking tendencies attend abroad-specific alcohol prevention programs prior to studying abroad.

We found similar results for sexual assault programs specifically tailored to students studying abroad. Students who said that they did not complete a study abroad-specific sexual assault program reported heavier drinking pre-departure and stronger intentions to drink while abroad compared to students who completed a study abroad-specific program. Because heavy drinking is a risk factor for sexual assault and other negative outcomes (Abbey, Zawacki, Buck, Clinton, \& McAuslan, 2004; Wechsler, Moeykens, Davenport, Castillo, \& Hansen, 1995), including while abroad (Flack et al., 2015), these results suggest that students most at risk are not receiving or remembering the information that might help them avoid dangerous situations. While pre-departure programs may be reaching fewer students at risk for alcohol misuse than students who do not report alcohol misuse, most students who reported attending a study abroad-specific sexual violence program indicated that alcohol use was discussed as a risk factor for sexual assault, which is promising.

Finally, there were significant differences in reported completion of abroad-specific alcohol and drug misuse programs and sexual assault programs based on school size. Compared to students studying at small schools, a greater proportion of participants from the medium and large schools reported completing an alcohol or drug program specific to going abroad. This result may reflect variation in resources across schools as bigger schools may be more able to provide programs, as highlighted by a greater proportion of students in large schools reporting receipt of an abroad- 
specific alcohol or drug misuse prevention programs. Low-cost online programming could provide one solution for smaller institutions if cost does represent a significant barrier to providing programs.

However, students at larger schools were less likely to complete abroad-specific sexual assault programs than those at smaller schools. While it is unclear why sexual assault prevention programs would have a smaller attendance than alcohol and drug misuse programs for students going abroad, lower attendance at larger schools could indicate that while larger schools have resources for prevention programming, students from large schools may more easily skip such programs without notice. For smaller schools that send only a small number of students abroad, it may be easier to keep track of attendance at mandatory pre-departure programs.

Increasing attendance at abroad-specific programs aimed at preventing alcohol and drug misuse and sexual assault is critical. Program directors might improve attendance in two ways. If students are able to avoid attending programming for alcohol and drug misuse prevention and sexual assault risk prevention without notice (as could be the case with larger schools), providing incentives for participation in offered programs, ensuring that they are mandatory, and documenting attendance may be practical solutions to encourage participation. However, if costs are an issue (as may be the case at small schools with smaller endowments and funding), lower-cost, online programming could provide an alternative solution to in-person prevention courses. It should be noted, nevertheless, that the differences between reported completion rates based on school size were small, reinforcing the need for increasing completion rates across schools of all sizes.

\section{Limitations}

One limitation of the study is that students may have received programming after they took the survey. However, participants completed surveys at most two weeks before their departure date and many completed it a few days before they left or within the first few days of arrival abroad. Still, some programs may include informational programming upon arrival to the country and it is possible that alcohol or drugs and sexual assault risks were discussed more fully with students once they were overseas. Further research is needed to understand whether program directors are relying on programming on-site instead of providing programming before departure at their home institution. In addition, it is important to note that the results of this survey represent students' reports of completed programming. It is possible that program directors and institutions offered the programs, but students failed to attend or did not remember attending. It may also be that students forgot the programing by the time they took the survey. If this is the case, however, this suggests a need for study abroad programs to be engaging enough to remain in students' minds when they travel abroad. Even if institutions offer programming, if students cannot recall the content, they are unlikely to have a significant impact.

\section{Conclusion and Future Directions}

Overall, this research suggests that although the majority of students who study abroad report completing alcohol or drug and sexual assault prevention programming in college, only about twofifths of students reported completing an alcohol or drug program specific to study abroad and only about one-fifth reported completing sexual assault programming specific to study abroad. Despite study abroad directors reporting they are delivering these programs to students (Marcantonio, 
2018b), our survey of students in this study and prior work examining school-specific website information from study abroad programs (Marcantonio et al., 2018c) indicate that students may not be receiving abroad-specific risk information. There is a missed opportunity to better prepare students for potential risks if institutions are not providing programming that specifically details these abroad-specific risks.

Given this evidence, there is a need for programs that target the unique and increased risks for students abroad and communicate this information in an engaging way so students can recall it for an extended period. A new pilot program seeks to prevent heavy alcohol use and resulting consequences among study abroad students (Pedersen, Neighbors, Atkins, Lee, \& Larimer, 2017; Pedersen et al., 2019). Using brief online personalized feedback interventions, the program aims to prevent increased and problematic use by correcting misperceptions of study abroad student drinking norms, correcting misperceptions of country-specific drinking norms, and promoting positive adjustment and engagement into the host culture prior to departure abroad, factors that have been identified in the research literature as serving as risk and protective factors for drinking abroad (Mitchell, Poyrazli, \& Broyles, 2016; Pedersen, Cruz, LaBrie, \& Hummer, 2011; Pedersen, LaBrie, \& Hummer, 2009; Pedersen et al., 2012). If the program is successful, it will represent an additional tool for program directors to consider when determining how to best deliver tailored programing to reduce alcohol consumption and lower students' risk of consequences of heavy drinking and sexual assault while they are away from home.

\section{Acknowledgements}

This work was funded by a grant from the National Institute on Alcohol Abuse and Alcoholism (R01AA025909, “Online Intervention to Prevent Risky Behaviors During College Student Study Abroad Experiences") awarded to Eric R. Pedersen. NIAAA had no role in the study design, collection, analysis or interpretation of the data, writing the manuscript, or the decision to submit the paper for publication.

\section{References}

Abbey, A., Zawacki, T., Buck, P. O., Clinton, A. M., \& McAuslan, P. (2004). Sexual assault and alcohol consumption: What do we know about their relationship and what types of research are still needed? Aggression and Violent Behavior, 9(3), 271-303. doi:10.1016/S1359-1789(03)00011-9

Aresi, G., Moore, S., \& Marta, E. (2016). Drinking, drug use, and related consequences among university students completing study abroad experiences: A systematic review. Substance Use and Misuse, 51(14), 1888-1904. doi:10.1080/10826084.2016.1201116

Collins, R. L., Parks, G. A., \& Marlatt, G. A. (1985). Social determinants of alcohol consumption: The effects of social interaction and model status on the self-administration of alcohol. Journal of Consulting and Clinical Psychology, 53(2), 189-200.

Fisher, B. S., Daigle, L. E., \& Cullen, F. T. (2009). Unsafe in the ivory tower: The sexual victimization of college women. Thousand Oaks, CA: Sage Publications.

Flack Jr., W. F., Kimble, M. O., Campbell, B. E., Hopper, A. B., Petercă, O., \& Heller, E. J. (2015). Sexual assault victimization among female undergraduates during study abroad: A single campus survey study. Journal of Interpersonal Violence, 30(20), 3453-3466. doi:10.1177/0886260514563833

Hummer, J. F., Pedersen, E. R., Mirza, T., \& Labrie, J. W. (2010). Factors associated with general and sexual alcohol-related consequences: An examination of college students studying abroad. Journal of Student Affairs Research and Practice, 47(4), 421-438. doi:10.2202/1949-6605.6134

Institute of International Education. (2018). Open Doors fast facts. Retrieved from https://www.iie.org/Research-and-Insights/Open-Doors/Fact-Sheets-and-Infographics/Fast-Facts 
Kimble, M., Flack Jr., W. F., \& Burbridge, E. (2013). Study abroad increases risk for sexual assault in female undergraduates: A preliminary report. Psychological Trauma: Theory, Research, Practice, and Policy, 5(5), 426-430. doi:10.1037/a0029608

Marcantonio, T., Angelone, D., \& Joppa, M. (2018a). Understanding contributing factors to verbal coercion while studying abroad. Journal of American College Health, 66(6), 440-444. doi:10.1080/07448481.2018.1431912

Marcantonio, T. L., Angelone, D., \& Sledjeski, E. (2016). Using a pattern-centered approach to assess sexual risk-taking in study abroad students. Journal of American College Health, 64(3), 165-173. doi:10.1080/07448481.2015.1085058

Marcantonio, T. L., Angelone, D., Swirsky, J., \& Joppa, M. (2018b). An analysis of the sexual health and safety information study abroad directors present their students prior to departure. Journal of American College Health, 1-5. doi:10.1080/07448481.2018.1515758

Marcantonio, T. L., Swirsky, J., Angelone, D. J., Joppa, M., \& Jozkowski, K. N. (2018c). A content analysis of sexual health and substance use information presented on study abroad websites: Findings and recommendations. Journal of American College Health, 1-9. doi:10.1080/07448481.2018.1499651

Mitchell, M. A., Poyrazli, S., \& Broyles, L. M. (2016). Hazardous alcohol use and cultural adjustment among US college students abroad in Italy: Findings and recommendations for study abroad staff and researchers. Substance Abuse, 37(1), 215-221. doi:10.1080/08897077.2015.1019663

Pedersen, E. R., Cruz, R. A., LaBrie, J. W., \& Hummer, J. F. (2011). Examining the relationships between acculturation orientations, perceived and actual norms, and drinking behaviors of short-term American sojourners in foreign environments. Prevention Science, 12, 401-410. doi:10.1007/s11121011-0232-7

Pedersen, E. R., D’Amico, E. J., LaBrie, J. W., Farris, C., Klein, D. J., \& Griffin, B. A. (2019). An online alcohol and risky sex prevention program for college students studying abroad: Study protocol for a randomized controlled trial. Addiction Science \& Clinical Practice, 14(32). doi:10.1186/s13722-019$0162-4$

Pedersen, E. R., LaBrie, J. W., \& Hummer, J. F. (2009). Perceived behavioral alcohol norms predict drinking for college students while studying abroad. Journal of Studies on Alcohol and Drugs, 70, 924-928.

Pedersen, E. R., Larimer, M. E., \& Lee, C. M. (2010). When in Rome: Factors associated with changes in drinking behavior among American college students studying abroad. Psychology of Addict Behaviors, 24(3), 535-540. doi:10.1037/a0019863

Pedersen, E. R., Neighbors, C., Atkins, D. C., Lee, C. M., \& Larimer, M. E. (2017). Brief online interventions targeting risk and protective factors for increased and problematic alcohol use among American college students studying abroad. Psychology of Addictive Behaviors, 31(2), 220-230. doi:10.1037/adb0000242

Pedersen, E. R., Neighbors, C., Lee, C. M., \& Larimer, M. E. (2012). Not all those who wander are lost: Examining the impact of sojourner adjustment and drinking motives on alcohol consequences experienced by Americans studying in foreign countries. Journal of Studies on Alcohol and Drugs, 73(6), 1005-1015.

Wechsler, H., Moeykens, B., Davenport, A., Castillo, S., \& Hansen, J. (1995). The adverse impact of heavy episodic drinkers on other college students. Journal of Studies on Alcohol, 56(6), 628-634. 